\title{
Kinetics of Triscarbonato Uranyl Reduction by Aqueous Ferrous Iron:
}

\section{A Theoretical Study}

Matthew C. F. Wander ${ }^{1,2}$, Sebastien Kerisit ${ }^{3}$, Kevin M. Rosso ${ }^{3}$, Martin A. A. Schoonen ${ }^{1,2}$

${ }^{1}$ Department of Geosciences, Stony Brook University, Stony Brook, NY 11794-2100

${ }^{2}$ Center for Environmental and Molecular Science (CEMS), Stony Brook University

${ }^{3}$ Pacific Northwest National Laboratory (PNNL), Richland, WA

\section{SUPPORTING INFORMATION}

Table 1. Potential parameters used in this work to model molecular water, uranyl, iron, and carbonate ions.

\begin{tabular}{|c|c|c|c|c|}
\hline \multicolumn{5}{|c|}{ Charges and core-shell spring constants ${ }^{\mathrm{a}}$} \\
\hline Ion & Core $(e)$ & Shell (e) & \multicolumn{2}{|c|}{$\mathrm{k}\left(\mathrm{eV} . \AA^{-2}\right)$} \\
\hline Uranium (U) & +2.500 & - & \multicolumn{2}{|c|}{-} \\
\hline Uranyl O (OU $)$ & +0.500 & -0.750 & \multicolumn{2}{|c|}{27.40} \\
\hline Iron $(\mathrm{Fe})$ & +2.000 & - & \multicolumn{2}{|c|}{-} \\
\hline Carbon $(\mathrm{C})$ & +1.135 & - & \multicolumn{2}{|c|}{-} \\
\hline Carbonate $\mathrm{O}\left(\mathrm{O}_{\mathrm{C}}\right)$ & +0.587 & -1.632 & \multicolumn{2}{|c|}{507.40} \\
\hline Water $\mathrm{O}\left(\mathrm{O}_{\mathrm{W}}\right)$ & +1.250 & -2.050 & \multicolumn{2}{|c|}{209.45} \\
\hline Hydrogen $(\mathrm{H})$ & +0.400 & - & \multicolumn{2}{|c|}{-} \\
\hline \multicolumn{5}{|c|}{ Buckingham potential parameters ${ }^{\mathrm{b}}$} \\
\hline Ion pair (ij) & $\mathrm{A}_{\mathrm{ij}}(\mathrm{eV})$ & $\rho_{\mathrm{ij}}(\AA)$ & \multicolumn{2}{|c|}{$\mathrm{C}_{\mathrm{ij}}\left(\mathrm{eV} . \AA^{6}\right)$} \\
\hline $\mathrm{Fe}-\mathrm{O}_{\mathrm{C}}$ & 420.00 & 0.3299 & \multicolumn{2}{|c|}{0.00} \\
\hline $\mathrm{C}-\mathrm{O}_{\mathrm{W}}$ & 435.00 & 0.3400 & \multicolumn{2}{|c|}{0.00} \\
\hline $\mathrm{C}-\mathrm{O}_{\mathrm{U}}$ & 435.00 & 0.3400 & \multicolumn{2}{|c|}{0.00} \\
\hline $\mathrm{O}_{\mathrm{C}}-\mathrm{O}_{\mathrm{C}}$ & 16372.00 & 0.2130 & \multicolumn{2}{|c|}{3.47} \\
\hline \multicolumn{5}{|c|}{ Born-Mayer potential parameters ${ }^{\mathrm{c}}$} \\
\hline Ion pair (ij) & $\mathrm{A}_{\mathrm{ij}}(\mathrm{eV})$ & $\mathrm{B}_{\mathrm{ij}}(\AA)$ & $\mathrm{C}_{\mathrm{ij}}\left(\mathrm{eV} . \AA^{12}\right)$ & $\mathrm{D}_{\mathrm{ij}}\left(\mathrm{eV} . \AA^{6}\right)$ \\
\hline $\mathrm{Fe}-\mathrm{Ow}$ & 45.00 & 0.5764 & 2200.00 & 75.00 \\
\hline \multicolumn{5}{|c|}{ Lennard-Jones potential parameters ${ }^{\mathrm{d}}$} \\
\hline Ion pair (ij) & $\mathrm{A}_{\mathrm{ij}}\left(\mathrm{eV} . \AA^{\mathrm{n}}\right)$ & $\mathrm{B}_{\mathrm{ij}}\left(\mathrm{eV} . \AA^{\mathrm{m}}\right)$ & $\mathrm{n}$ & $\mathrm{m}$ \\
\hline $\mathrm{U}-\mathrm{O}_{\mathrm{C}}$ & 15000.00 & 22.00 & 12 & 6 \\
\hline $\mathrm{U}-\mathrm{O}_{\mathrm{W}}$ & 15000.00 & 22.00 & 12 & 6 \\
\hline $\mathrm{O}_{\mathrm{U}}-\mathrm{O}_{\mathrm{C}}$ & 27291.72 & 27.12 & 12 & 6 \\
\hline $\mathrm{O}_{\mathrm{U}}-\mathrm{O}_{\mathrm{W}}$ & 27291.72 & 27.12 & 12 & 6 \\
\hline $\mathrm{O}_{\mathrm{C}}-\mathrm{O}_{\mathrm{W}}$ & 27291.72 & 27.12 & 12 & 6 \\
\hline Ow-Ow & 39344.98 & 42.15 & 12 & 6 \\
\hline $\mathrm{H}-\mathrm{Ow}$ & 24.00 & 6.00 & 9 & 6 \\
\hline
\end{tabular}


Table I. (cont.)

\begin{tabular}{|c|c|c|c|c|}
\hline \multicolumn{5}{|c|}{ Morse potential parameters ${ }^{\mathrm{e}}$} \\
\hline Ion pair (ij) & $D_{i j}(e V)$ & $\alpha_{i j}\left(\AA^{-1}\right)$ & $\mathrm{r}_{0}(\AA)$ & Coul. Sub. (\%) \\
\hline $\mathrm{C}-\mathrm{O}_{\mathrm{C}}$ & 4.7100 & 3.8000 & 1.1800 & 0 \\
\hline $\mathrm{Ow}-\mathrm{H}$ & 6.2037 & 2.2200 & 0.9237 & 50 \\
\hline $\mathrm{H}-\mathrm{H}$ & 0.0000 & 2.8405 & 1.5000 & 50 \\
\hline \multicolumn{5}{|c|}{ Harmonic potential parameters ${ }^{\mathrm{f}}$} \\
\hline Ions (ijk) & \multicolumn{2}{|c|}{$\mathrm{k}_{\mathrm{ij}}\left(\mathrm{eV} . \AA^{-\frac{1}{2}}\right)$} & \multicolumn{2}{|r|}{$\mathrm{r}_{0}$} \\
\hline $\mathrm{U}-\mathrm{O}_{\mathrm{U}}$ & \multicolumn{4}{|c|}{ Three-body potential parameters ${ }^{\mathrm{g}}$} \\
\hline Ions (ijk) & \multicolumn{3}{|c|}{$\mathrm{k}_{\mathrm{ijk}}\left(\mathrm{eV} \cdot \mathrm{rad}^{-2}\right)$} & $\theta_{0}$ \\
\hline $\mathrm{O}_{\mathrm{C}}-\mathrm{C}-\mathrm{O}_{\mathrm{C}}$ & \multicolumn{3}{|c|}{1.6900} & 120.00 \\
\hline $\mathrm{O}_{\mathrm{U}}-\mathrm{U}-\mathrm{O}_{\mathrm{U}}$ & \multicolumn{3}{|c|}{13.009} & 180.00 \\
\hline $\mathrm{H}-\mathrm{Ow}-\mathrm{H}$ & & & & 108.69 \\
\hline & \multicolumn{4}{|c|}{ Four-body potential parameters ${ }^{\mathrm{h}}$} \\
\hline Ions (ijkl) & \multicolumn{3}{|c|}{$\mathrm{k}_{\mathrm{ijkl}}\left(\mathrm{eV} \cdot \mathrm{rad}^{-1}\right)$} & $\theta_{0}$ \\
\hline $\mathrm{O}_{\mathrm{C}}-\mathrm{C}-\mathrm{O}_{\mathrm{C}}-\mathrm{O}_{\mathrm{C}}$ & \multicolumn{2}{|c|}{0.1129} & & 180.00 \\
\hline $\mathrm{O}_{\mathrm{C}}-\mathrm{U}-\mathrm{O}_{\mathrm{C}}-\mathrm{O}_{\mathrm{C}}$ & \multicolumn{3}{|c|}{0.5000} & 180.00 \\
\hline
\end{tabular}

a. Spring constant potential form: $\mathrm{V}=\mathrm{k} \cdot \mathrm{r}_{\mathrm{c}-\mathrm{s}}{ }^{2}$

b. Buckingham potential form: $\mathrm{V}_{\mathrm{ijj}}=\mathrm{A}_{\mathrm{ij}} \exp \left(-\mathrm{r}_{\mathrm{ij}} / \rho_{\mathrm{ij}}\right)-\mathrm{C}_{\mathrm{ij}} \mathrm{r}_{\mathrm{ij}}{ }^{-6}$

c. Born-Mayer potential form: $\mathrm{V}_{\mathrm{ij}}=\mathrm{A}_{\mathrm{ij}} \exp \left(-\mathrm{r}_{\mathrm{ij}} / \mathrm{B}_{\mathrm{ij}}\right)+\mathrm{C}_{\mathrm{ij}} \mathrm{r}_{\mathrm{ij}}{ }^{-12}-\mathrm{D}_{\mathrm{ij}} \mathrm{r}_{\mathrm{ij}}{ }^{-6}$

d. Lennard-Jones potential form: $\mathrm{V}_{\mathrm{ij}}=\mathrm{A}_{\mathrm{ij}} \mathrm{r}_{\mathrm{ij}}{ }^{-}{ }^{-}-\mathrm{B}_{\mathrm{ijj}} \mathrm{r}_{\mathrm{ij}}{ }^{-\mathrm{m}}$

e. Morse potential form: $V_{\mathrm{ij}}=D_{\mathrm{ij}}\left(1-\exp \left[-\alpha_{\mathrm{ij}}\left(\mathrm{r}_{\mathrm{ij}}-\mathrm{r}_{0}\right)\right]^{2}\right)-\mathrm{D}_{\mathrm{ij}}$

f. Harmonic potential form: $\mathrm{V}_{\mathrm{ij}}=\mathrm{k}_{\mathrm{ij}}\left(\mathrm{r}_{\mathrm{ij}}-\mathrm{r}_{0}\right)^{2}$.

g. Three-body potential form: $\mathrm{V}_{\mathrm{ijk}}=0.5 \cdot \mathrm{k}_{\mathrm{ijk}}\left(\theta_{\mathrm{ijk}}-\theta_{0}\right)^{2}$.

h. Four-body potential form: $\mathrm{V}_{\mathrm{ijkl}}=\mathrm{k}_{\mathrm{ijkk}}\left[1+\cos \left(2 \theta_{\mathrm{ijkl}}-\theta_{0}\right)\right]$. 
Table II. Comparison of the structural parameters of the uranyl ion in aqueous solution obtained in this work with published experimental and theoretical data.

\begin{tabular}{|c|c|c|c|c|}
\hline Source & $\begin{array}{l}R_{l s t} \mathrm{U}-\mathrm{O}_{\mathrm{H} 2 \mathrm{O}} \\
(\AA)\end{array}$ & $n$ & $\begin{array}{c}R_{2 n d} \mathrm{U}_{-} \mathrm{O}_{\mathrm{H} 2 \mathrm{O}} \\
(\AA)\end{array}$ & Method/Model \\
\hline This work & 2.45 & 5.0 & 4.68 & $\begin{array}{l}\text { Modified Guilbaud and Wipff } \text { (uranyl) }^{\text {a }} \text { (e Leeuw and Parker }{ }^{\mathrm{b}} \text { (water) } \\
\text { de }\end{array}$ \\
\hline Guilbaud and Wipff ${ }^{\mathrm{a}}$ & 2.50 & 5.0 & - & $\begin{array}{c}\text { Guilbaud and Wipff }{ }^{\mathrm{a}} \text { (uranyl) } \\
\text { TIP3P (water) }\end{array}$ \\
\hline Hagberg et $a .^{\mathrm{c}}$ & 2.40 & 5.0 & 4.70 & $\begin{array}{l}\text { Hagberg et al. }{ }^{\mathrm{c}} \text { (uranyl) } \\
\text { Brdarski et al. }{ }^{\mathrm{d}} \text { (water) }\end{array}$ \\
\hline Greathouse et al. ${ }^{\mathrm{e}}$ & 2.49 & 5.0 & 4.85 & $\begin{array}{l}\text { Guilbaud and Wipff (uranyl) } \\
\text { Flexible SPC (water) }\end{array}$ \\
\hline Allen et $a l .{ }^{\mathrm{f}}$ & 2.41 & 5.3 & - & $\begin{array}{l}\text { XAFS - aqueous solution } \\
\text { low chloride concentration }\end{array}$ \\
\hline Sémon et al. ${ }^{\mathrm{g}}$ & 2.40 & 4.6 & - & $\begin{array}{l}\text { EXAFS - aqueous solution } \\
\text { low perchlorate concentration }\end{array}$ \\
\hline Wahlgren et al. ${ }^{\mathrm{h}}$ & 2.41 & 4.5 & - & $\begin{array}{l}\text { EXAFS - aqueous solution } \\
\text { low perchlorate concentration }\end{array}$ \\
\hline Neuefeind et $a l^{i}$ & 2.42 & 5.0 & 4.46 & $\begin{array}{l}\text { XRS - aqueous solution } \\
\text { low perchlorate concentration }\end{array}$ \\
\hline Åberg et al. ${ }^{\mathrm{j}}$ & 2.42 & 5.0 & 4.50 & XRD- aqueous perchlorate solution \\
\hline
\end{tabular}

a. Guilbaud, P.; Wipff, G. J. Mol. Struct. (THEOCHEM) 1996, 366, 55.

b. De Leeuw, N.H.; Parker, S.C. Phys. Rev. B 1998, 58, 13901.

c. Hagberg, D.; Karlström, G.; Ross, B.O.; Gagliardi, L. J. Am. Chem. Soc. 2005, 127, 14250.

d. Brdarski, S.; Åstrand, P.; Karlström, G. Theor. Chem. Acc. 2000, 105, 7.

e. Greathouse, J.A.; O’Brien, R.J.; Bemis, G.; Pabalan, R.T. J. Phys. Chem. B 2002, 106, 1646.

f. $\quad$ Allen, P.G.; Bucher, J.J.; Shuh, D.K.; Edelstein, N.M.; Reich, T. Inorg. Chem. 1997, 36, 4676.

g. Sémon, L.; Boehme, C.; Billard, I.; Hennig, C.; Lützenkrichen, K.; Reich, T.; Roßberg, A.; Rossini, I.; Wipff, G. ChemPhysChem 2001, 2, 591.

h. Wahlgren, U.; Moll, H.; Grenthe, I.; Schimmelpfennig, B.; Maron, L.; Vallet, V.; Gropen O. Inorg. Chem. 2001, 40, 3516.

i. $\quad$ Neuefeind, J.; Soderholm, L.; Skanthakumar, S. J. Phys. Chem. A 2004, 108, 2733.

j. Åberg, M.; Ferri, D.; Glaser, J.; Grenthe, I. Inorg. Chem. 1983, 22, 3986. 
Figure 1. Uranyl oxygen-water oxygen and uranyl oxygen-water hydrogen radial distribution functions showing the lack of direct hydrogen bonding between water and uranyl oxygen ions and the formation of a $3 \AA$ cavity around uranyl oxygen ions as described by Hagberg et al. ${ }^{\text {. }}$.

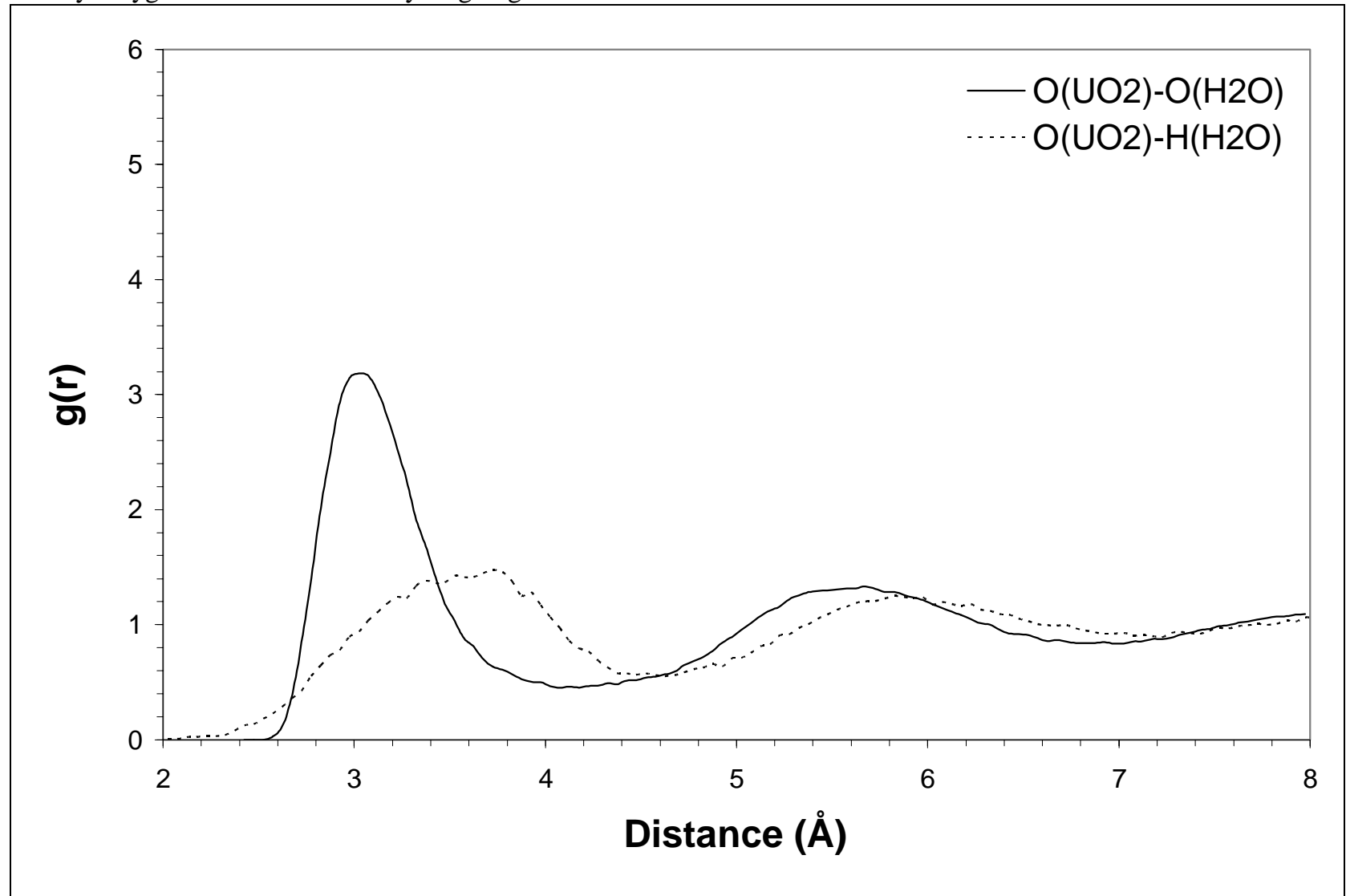

${ }^{a}$ Hagberg, D.; Karlström, G.; Ross, B.O.; Gagliardi, L. J. Am. Chem. Soc. 2005, 127, 14250. 
Table III. Comparison of the structural parameters $(\AA)$ of the triscarbonato uranyl complex obtained in this work with published experimental and theoretical data.

\begin{tabular}{|c|c|c|c|c|c|}
\hline Source & $R \mathrm{U}-\mathrm{O}_{1}$ & $R \mathrm{U}-\mathrm{O}_{2}$ & $R \mathrm{U}-\mathrm{O}_{3}$ & $R \mathrm{U}-\mathrm{C}$ & Method/Model \\
\hline This work & 1.83 & 2.44 & 4.06 & 2.86 & gas-phase \\
\hline This work & 1.82 & 2.39 & 3.97 & 2.80 & solution \\
\hline Hemmingsen et al. ${ }^{\mathrm{a}}$ & 1.85 & 2.58 & - & - & B3LYP/DZ - gas-phase \\
\hline Hemmingsen et al. ${ }^{\mathrm{a}}$ & 1.77 & 2.57 & - & - & HF/DZ - gas-phase \\
\hline Vázquez et $a l .^{\text {b }}$ & 1.85 & 2.56 & 4.29 & 3.01 & DFT - gas-phase \\
\hline Vázquez et al. ${ }^{\mathrm{b}}$ & 1.85 & 2.46 & 4.16 & 2.90 & DFT - solution \\
\hline Gagliardi et $a{ }^{\mathrm{c}}{ }^{\mathrm{c}}$ & 1.89 & 2.43 & 4.79 & 2.89 & MBPT2 - gas-phase \\
\hline Gagliardi et $a .^{\mathrm{c}}$ & 1.88 & 2.41 & 4.11 & 2.84 & MBPT2 - solution \\
\hline Bernhard et al. ${ }^{\mathrm{d}}$ & 1.80 & 2.44 & 4.20 & 2.89 & EXAFS \\
\hline Docrat et al. ${ }^{\mathrm{e}}$ & 1.80 & 2.43 & 4.13 & 2.81 & EXAFS \\
\hline
\end{tabular}

a. Hemmingsen, L.; Amara, P.; Ansoborlo, E; Field, M.J. J. Phys. Chem. A 2000, 104, 4095.

b. Vázquez, J.; Bo, C.; Poblet, J.M.; de Pablo, J.; Bruno, J. Inorg. Chem. 2003, 42, 6136.

c. Gagliardi, L.; Grenthe, I.; Ross, B.O. Inorg. Chem. 2001, 40, 2976.

d. Bernhard, G.; Geipel, G.; Reich, T.; Brendler, V.; Amayri, S.; Nitsche, H. Radiochim. Acta 2001, 89, 511.

e. Docrat, T.I.; Mosselmans, J.F.W.; Charnock, J.M.; Whiteley, M.; Collison, D.; Livens, R.F.; Jones, C.; Edmiston, M. Inorg. Chem. 1999, 38, 1879. 
Figure 2. Snapshots from the MD simulations of the 2xmonodentate (a), 1xbidentate (b), solvent-separated (c), and 1xmonodentate (d) iron(II)-triscarbonato uranyl complexes.

a

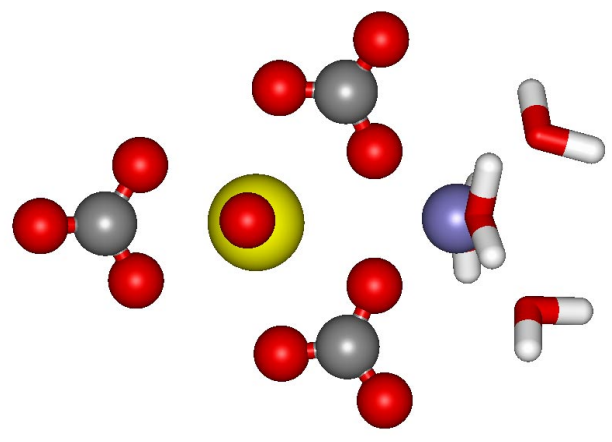

$\mathrm{c}$

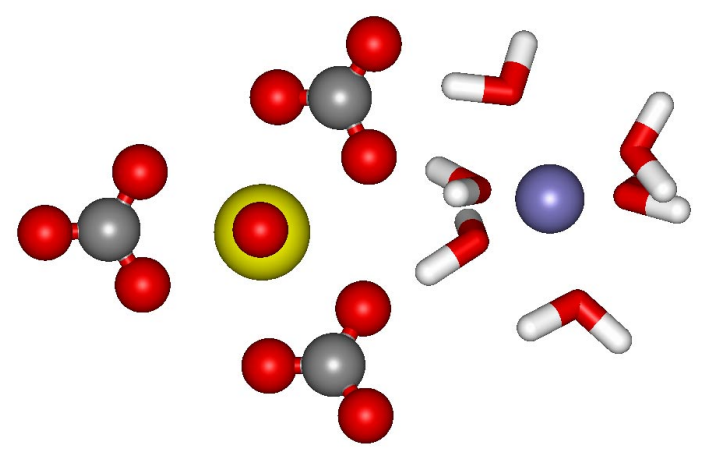

b

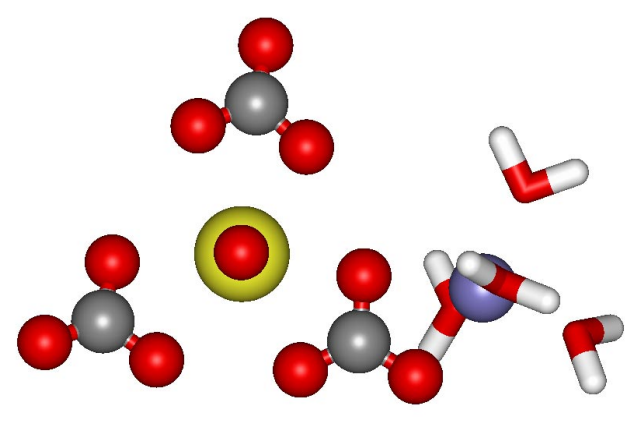

d

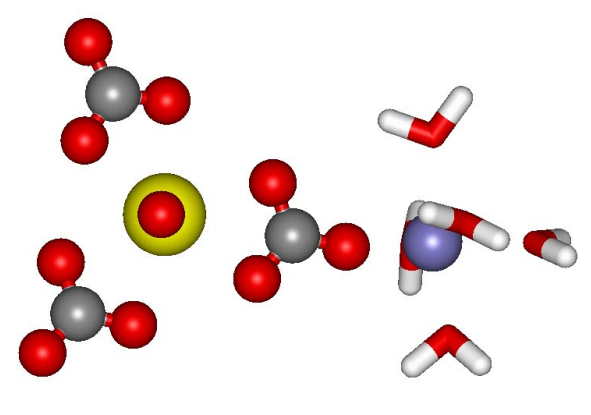


Table IV. Comparison of the distances $(\AA)$ calculated from the potential model and DFT calculations for the II/II/IV iron triscarbonato uranyl complex.

\begin{tabular}{ccccccc}
\hline Distance & $\mathrm{Fe}_{1}-\mathrm{O}_{\mathrm{H} 2 \mathrm{O}}$ & $\mathrm{Fe}_{1}-\mathrm{O}_{\mathrm{H} 2 \mathrm{O}}$ & $\mathrm{Fe}_{1}-\mathrm{O}_{\mathrm{H} 2 \mathrm{O}}$ & $\mathrm{Fe}_{1}-\mathrm{O}_{\mathrm{H} 2 \mathrm{O}}$ & $\mathrm{Fe}_{1}-\mathrm{O}_{\mathrm{CO}}$ & $\mathrm{Fe}_{1}-\mathrm{O}_{\mathrm{CO}}$ \\
$\mathrm{MD}$ & 2.28 & 2.07 & 2.38 & 2.04 & 2.28 & 1.82 \\
DFT & 2.34 & 2.22 & 2.16 & 1.95 & 2.76 & 2.01 \\
\hline Distance & $\mathrm{Fe}_{2}-\mathrm{O}_{\mathrm{H} 2 \mathrm{O}}$ & $\mathrm{Fe}_{2}-\mathrm{O}_{\mathrm{H} 2 \mathrm{O}}$ & $\mathrm{Fe}_{2}-\mathrm{O}_{\mathrm{H} 2 \mathrm{O}}$ & $\mathrm{Fe}_{2}-\mathrm{O}_{\mathrm{H} 2 \mathrm{O}}$ & $\mathrm{Fe}_{2}-\mathrm{O}_{\mathrm{CO}}$ & $\mathrm{Fe}_{2}-\mathrm{O}_{\mathrm{CO}}$ \\
MD & 2.18 & 2.16 & 2.11 & 2.26 & 1.92 & 1.84 \\
DFT & 2.30 & 2.11 & 2.20 & 2.28 & 2.09 & 2.02 \\
\hline Distance & $\mathrm{U}-\mathrm{O}_{\mathrm{CO}}$ & $\mathrm{U}-\mathrm{O}_{\mathrm{CO}}$ & $\mathrm{U}-\mathrm{O}_{\mathrm{CO}}$ & $\mathrm{U}-\mathrm{O}_{\mathrm{CO}}$ & $\mathrm{U}-\mathrm{O}_{\mathrm{CO}}$ & \\
MD & 2.41 & 2.31 & 2.31 & 2.43 & 2.36 & \\
DFT & 2.45 & 2.36 & 2.35 & 2.43 & 2.49 & \\
\hline Distance & $\mathrm{U}-\mathrm{O}_{\mathrm{UO}}$ & $\mathrm{U}-\mathrm{O}_{\mathrm{UO}}$ & $\mathrm{Fe}$ & & & \\
MD & 1.82 & 1.82 & 3.72 & 4.04 & & \\
DFT & 1.78 & 1.78 & 3.86 & 4.07 & & \\
\hline
\end{tabular}


Table V. Comparison of the experimental and calculated cell parameters and bulk modulus of siderite $\left(\mathrm{FeCO}_{3}\right)$.

\begin{tabular}{lcccc}
\hline Source & $\mathrm{a}(\AA)$ & $\mathrm{b}(\AA)$ & $\mathrm{c}(\AA)$ & Bulk modulus $(\mathrm{GPa})$ \\
\hline Calculated & 4.4696 & 4.4696 & 15.3465 & 105 \\
Experimental $^{\mathrm{a}}$ & 4.6916 & 4.6916 & 15.3796 & $117^{\mathrm{b}}$ \\
\hline
\end{tabular}

a. Effenberger, H.; Mereiter, K.; Zemann, J. Z. Kristalogr. 1981, 156, 233.

b. Zhang, J.; Reeder, R.J. Am. Mineral. 1999, 84, 861. 
Table VI: Final Ab-intio optimized structures:

\begin{tabular}{|c|c|c|c|}
\hline \multicolumn{4}{|c|}{$\mathrm{Fe}(\mathrm{II}) \mathrm{Fe}(\mathrm{II}) \mathrm{U}(\mathrm{VI}) \mathrm{O}_{2}\left(\mathrm{CO}_{3}\right)_{2}\left(\mathrm{H}_{2} \mathrm{O}\right)_{8}$} \\
\hline U & 0.39047227 & -0.27937199 & -0.68307657 \\
\hline $\mathrm{O}$ & 0.26109998 & 1.37425051 & -0.03708733 \\
\hline $\mathrm{O}$ & 0.43130597 & -1.93320667 & -1.32585800 \\
\hline $\mathrm{Fe}$ & -1.44147978 & -0.16653943 & -4.08365861 \\
\hline $\mathrm{Fe}$ & -2.59082340 & -0.18439402 & 2.07889965 \\
\hline $\mathrm{C}$ & 0.37667567 & -1.08320329 & 2.06304414 \\
\hline $\mathrm{O}$ & 1.41690370 & -0.99571087 & 1.31969847 \\
\hline $\mathrm{O}$ & -0.76619070 & -0.92701608 & 1.38217332 \\
\hline $\mathrm{O}$ & 0.37082404 & -1.26655533 & 3.30030216 \\
\hline C & 1.44160912 & 0.44532387 & -3.24586446 \\
\hline $\mathrm{O}$ & 0.11439627 & 0.47062929 & -2.98224345 \\
\hline $\mathrm{O}$ & 2.12482318 & 0.29616865 & -2.16437388 \\
\hline $\mathrm{O}$ & 1.87217143 & 0.52208761 & -4.40208173 \\
\hline C & -3.19456172 & -0.20128028 & -0.94862168 \\
\hline $\mathrm{O}$ & -2.00252954 & -0.25611026 & -1.38133441 \\
\hline $\mathrm{O}$ & -3.50876584 & -0.09974400 & 0.28356571 \\
\hline $\mathrm{O}$ & -4.20358911 & -0.23888822 & -1.78992787 \\
\hline $\mathrm{O}$ & -3.37067564 & -0.43401238 & -4.18813939 \\
\hline $\mathrm{H}$ & -3.74575416 & -1.17765555 & -4.66759963 \\
\hline $\mathrm{H}$ & -3.88503971 & -0.35139125 & -2.76143169 \\
\hline $\mathrm{O}$ & -0.71070836 & -2.25716871 & -3.93467662 \\
\hline $\mathrm{H}$ & -0.06495020 & -2.51049714 & -4.60456031 \\
\hline $\mathrm{H}$ & -0.29376644 & -2.41535570 & -3.06797813 \\
\hline $\mathrm{O}$ & -0.23287164 & 0.10162351 & -5.85787490 \\
\hline $\mathrm{H}$ & -0.48466707 & 0.87685891 & -6.37172626 \\
\hline $\mathrm{H}$ & 0.66656518 & 0.31252051 & -5.44210960 \\
\hline $\mathrm{O}$ & -2.28984726 & 1.97638262 & -4.51096153 \\
\hline $\mathrm{H}$ & -2.25720262 & 2.66212867 & -3.83536064 \\
\hline $\mathrm{H}$ & -3.18546981 & 1.59797915 & -4.50344958 \\
\hline $\mathrm{O}$ & -1.67985227 & 1.81578091 & 1.99360224 \\
\hline $\mathrm{H}$ & -2.18874570 & 2.62838807 & 1.90839144 \\
\hline $\mathrm{H}$ & -0.95175357 & 1.85374340 & 1.34212908 \\
\hline $\mathrm{O}$ & -1.89089185 & -0.58035480 & 4.03258977 \\
\hline $\mathrm{H}$ & -1.85619374 & 0.10288258 & 4.70964823 \\
\hline $\mathrm{H}$ & -0.89556485 & -0.90157183 & 3.83503755 \\
\hline $\mathrm{O}$ & -4.66541564 & 0.61933701 & 2.56533579 \\
\hline $\mathrm{H}$ & -5.22780505 & 0.02284023 & 3.07296960 \\
\hline $\mathrm{H}$ & -4.95062209 & 0.53195096 & 1.63950841 \\
\hline 0 & -3.46380037 & -2.27187180 & 2.50342612 \\
\hline $\mathrm{H}$ & -2.99997820 & -2.51413513 & 3.31667059 \\
\hline $\mathrm{H}$ & -3.23823547 & -2.94392772 & 1.84935399 \\
\hline \multicolumn{4}{|c|}{$\mathrm{Fe}(\mathrm{III}) \mathrm{Fe}(\mathrm{II}) \mathrm{U}(\mathrm{V}) \mathrm{O}_{2}\left(\mathrm{CO}_{3}\right)_{2}\left(\mathrm{H}_{2} \mathrm{O}\right)_{8}$} \\
\hline $\mathrm{U}$ & 0.44436076 & -0.26668506 & -0.63083014 \\
\hline $\mathrm{O}$ & 0.23562612 & 1.42377684 & 0.03038336 \\
\hline $\mathrm{O}$ & 0.54549976 & -1.93334841 & -1.37030978 \\
\hline $\mathrm{Fe}$ & -1.49202907 & -0.16850862 & -3.96368517 \\
\hline $\mathrm{Fe}$ & -2.66222649 & -0.17226423 & 1.84455174 \\
\hline C & -0.00295894 & -1.42186252 & 2.00594122 \\
\hline $\mathrm{O}$ & 1.15724713 & -1.23640182 & 1.54383594 \\
\hline $\mathrm{O}$ & -0.99522324 & -1.00289388 & 1.17205759 \\
\hline
\end{tabular}




\begin{tabular}{|c|c|c|c|}
\hline $\mathrm{O}$ & -0.33603207 & -1.91370346 & 3.11687010 \\
\hline C & 1.45168270 & 0.52269285 & -3.30503251 \\
\hline $\mathrm{O}$ & 0.16253464 & 0.54614916 & -3.03879456 \\
\hline $\mathrm{O}$ & 2.25911689 & 0.46743271 & -2.37560003 \\
\hline $\mathrm{O}$ & 1.82400217 & 0.53759171 & -4.57068407 \\
\hline C & -3.25015747 & -0.25340168 & -1.27786072 \\
\hline $\mathrm{O}$ & -2.06915312 & -0.21742790 & -1.80708549 \\
\hline $\mathrm{O}$ & -3.48644326 & -0.20055851 & -0.05385435 \\
\hline $\mathrm{O}$ & -4.30881934 & -0.32991153 & -2.07698275 \\
\hline $\mathrm{O}$ & -3.31218319 & -0.36417617 & -4.45883014 \\
\hline $\mathrm{H}$ & -3.60225794 & -0.94560217 & -5.16734149 \\
\hline $\mathrm{H}$ & -4.02702279 & -0.40416826 & -3.03463752 \\
\hline $\mathrm{O}$ & -0.86422514 & -2.15691075 & -3.59217745 \\
\hline $\mathrm{H}$ & -0.29037981 & -2.47226521 & -4.30131778 \\
\hline $\mathrm{H}$ & -0.34399023 & -2.22439200 & -2.73536398 \\
\hline $\mathrm{O}$ & -0.54024853 & -0.16102495 & -5.60328725 \\
\hline $\mathrm{H}$ & -0.96103085 & 0.04442126 & -6.44352376 \\
\hline $\mathrm{H}$ & 1.01980608 & 0.40479685 & -5.14013095 \\
\hline $\mathrm{O}$ & -2.01502246 & 1.98552603 & -3.88573817 \\
\hline $\mathrm{H}$ & -1.57937122 & 2.44119637 & -3.15434829 \\
\hline $\mathrm{H}$ & -2.96636501 & 2.13221880 & -3.81621443 \\
\hline $\mathrm{O}$ & -1.86102816 & 1.90116026 & 1.65244351 \\
\hline $\mathrm{H}$ & -2.40006534 & 2.60647295 & 1.28061626 \\
\hline $\mathrm{H}$ & -1.04258644 & 1.84424485 & 1.08953154 \\
\hline $\mathrm{O}$ & -1.94591347 & 0.02815135 & 3.95112159 \\
\hline $\mathrm{H}$ & -1.46686212 & 0.85740131 & 4.06635554 \\
\hline $\mathrm{H}$ & -1.26206279 & -0.69380561 & 3.93161030 \\
\hline $\mathrm{O}$ & -4.50869724 & 0.45175147 & 2.84142449 \\
\hline $\mathrm{H}$ & -4.43193415 & 0.13725711 & 3.75275310 \\
\hline $\mathrm{H}$ & -5.32778604 & 0.09345053 & 2.47937840 \\
\hline $\mathrm{O}$ & -3.08137076 & -2.28049439 & 2.62578945 \\
\hline $\mathrm{H}$ & -2.14253967 & -2.45361076 & 2.87742312 \\
\hline $\mathrm{H}$ & -3.29479469 & -2.93135942 & 1.94789221 \\
\hline \multicolumn{4}{|c|}{$\mathrm{Fe}(\mathrm{III}) \mathrm{Fe}(\mathrm{III}) \mathrm{U}(\mathrm{IV}) \mathrm{O}_{2}\left(\mathrm{CO}_{3}\right)_{2}\left(\mathrm{H}_{2} \mathrm{O}\right)_{8}$} \\
\hline$U$ & 0.62385341 & -0.26455967 & -0.67721312 \\
\hline $\mathrm{O}$ & 0.23105381 & 1.71033741 & -0.06910104 \\
\hline $\mathrm{O}$ & 0.50657940 & -2.24104577 & -1.34617373 \\
\hline $\mathrm{Fe}$ & -1.46387773 & -0.42646123 & -3.68417255 \\
\hline $\mathrm{Fe}$ & -2.45547271 & 0.09703285 & 1.83678204 \\
\hline C & 0.37873549 & -0.90124009 & 2.18349523 \\
\hline $\mathrm{O}$ & 1.44961257 & -0.88131880 & 1.51154926 \\
\hline $\mathrm{O}$ & -0.73820395 & -0.69700476 & 1.40788424 \\
\hline $\mathrm{O}$ & 0.24210092 & -1.07923269 & 3.40869021 \\
\hline C & 1.46829108 & 0.30605566 & -3.41133143 \\
\hline $\mathrm{O}$ & 0.15936227 & 0.38817785 & -2.97507447 \\
\hline $\mathrm{O}$ & 2.27148993 & 0.19622295 & -2.43884093 \\
\hline $\mathrm{O}$ & 1.68699464 & 0.33485436 & -4.63093412 \\
\hline C & -3.12649184 & -0.03446551 & -1.17599242 \\
\hline $\mathrm{O}$ & -1.94621982 & -0.36573524 & -1.64458593 \\
\hline $\mathrm{O}$ & -3.33519519 & -0.15452214 & 0.09767441 \\
\hline $\mathrm{O}$ & -4.04261375 & 0.37422309 & -1.93736790 \\
\hline $\mathrm{O}$ & -3.50090367 & -0.65326758 & -4.22124696 \\
\hline
\end{tabular}




\begin{tabular}{llll}
\hline $\mathrm{H}$ & -3.73342064 & -1.58358109 & -4.32329488 \\
$\mathrm{H}$ & -3.94969287 & -0.29667671 & -3.39132971 \\
$\mathrm{O}$ & -1.07414216 & -2.23179540 & -3.83370933 \\
$\mathrm{H}$ & -0.68832620 & -2.58221858 & -4.64431354 \\
$\mathrm{H}$ & 0.07337969 & -2.59979596 & -2.13943511 \\
$\mathrm{O}$ & -0.66526007 & 0.04885270 & -5.58056878 \\
$\mathrm{H}$ & -1.01649912 & 0.84011424 & -6.00497578 \\
$\mathrm{H}$ & 0.32945599 & 0.21597920 & -5.35953123 \\
$\mathrm{O}$ & -2.18767781 & 1.73278062 & -3.67978499 \\
$\mathrm{H}$ & -1.49703558 & 2.19312565 & -3.18260182 \\
$\mathrm{H}$ & -2.98467105 & 1.74470200 & -3.12049001 \\
$\mathrm{O}$ & -2.00415163 & 1.88815342 & 1.88828639 \\
$\mathrm{H}$ & -2.65275984 & 2.59926100 & 1.90665292 \\
$\mathrm{H}$ & -0.28894294 & 2.08067197 & 0.66266801 \\
$\mathrm{O}$ & -2.08537954 & -0.34129726 & 3.86616070 \\
$\mathrm{H}$ & -2.07834290 & 0.44571625 & 4.42342361 \\
$\mathrm{H}$ & -1.09139733 & -0.68864432 & 3.81222352 \\
$\mathrm{O}$ & -4.59482450 & 0.38591525 & 2.40006548 \\
$\mathrm{H}$ & -4.90059075 & -0.39105874 & 2.88578221 \\
$\mathrm{H}$ & -4.97315631 & 0.30971247 & 1.50918944 \\
$\mathrm{O}$ & -3.26282714 & -2.11394752 & 2.09704881 \\
$\mathrm{H}$ & -2.72322183 & -2.59599298 & 2.73656570 \\
$\mathrm{H}$ & -3.13051530 & -2.54711288 & 1.24427758 \\
& & & \\
\hline
\end{tabular}

